\title{
Protée
}

\section{Le formalisme russe}

\section{entre pensée organique allemande et premier structuralisme}

\section{Sergueï Tchougounnikov}

Volume 31, numéro 2, automne 2003

Cannes hors projections

URI : https://id.erudit.org/iderudit/008757ar

DOI : https://doi.org/10.7202/008757ar

Aller au sommaire du numéro

\section{Éditeur(s)}

Département des arts et lettres - Université du Québec à Chicoutimi

ISSN

0300-3523 (imprimé)

1708-2307 (numérique)

Découvrir la revue

\section{Citer cet article}

Tchougounnikov, S. (2003). Le formalisme russe : entre pensée organique allemande et premier structuralisme. Protée, 31(2), 83-98.

https://doi.org/10.7202/008757ar
Résumé de l'article

On examinera ici les « affinités électives " qui relient le modèle organique de l'« absolu littéraire » du romantisme allemand aux concepts linguistiques et poétiques du formalisme russe. Ce modèle, extrêmement important dans le contexte russe, est fondé sur la métaphore de l'organisme vivant qui fait écho aux théories de l'idéalisme allemand. Cette figure épistémologique est définie dans cette tradition organiciste comme un passage de la forme à la structure par le biais fonctionnel des organes. On trouve cet analogon organique au fondement du projet structuraliste (R. Jakobson) issu des écrits du formalisme russe et de la tradition organiciste dans le discours russe sur le langage et la littérature. 


\title{
LE FORMALISME RUSSE \\ ENTRE PENSÉE ORGANIQUE ALLEMANDE ET PREMIER STRUCTURALISME
}

\author{
SERGUEÏ TCHOUGOUNNIKOV
}

Plus mou est l'animal, plus dure est la carapace.

J.G. Herder

\section{Le modèle morphologique allemand}

Notre objectif est d'examiner la figure du modèle organique dans le discours russe sur le langage et sur la littérature tel qu'il s'est articulé entre le XIX et le XX $\mathrm{X}^{\mathrm{e}}$ siècle. Ce modèle, extrêmement important dans le contexte russe, est fondé sur la métaphore de l'organisme vivant, faisant écho aux théories de l'idéalisme allemand. Dans la tradition organiciste, cette figure épistémologique est définie comme un passage de la forme à la structure par le biais fonctionnel des organes ${ }^{1}$. On trouve cet analogon organique au fondement du projet formaliste et structuraliste russo-soviétique. En effet, cette métaphore de l'organisme réunit des penseurs aussi différents que Mikhail Bakhtine et son cercle et des théoriciens eurasistes comme N. Troubetzkoy et R. Jakobson. On peut aussi constater les effets de la même figure organique dans le discours marxiste, en particulier dans la doctrine officielle du "réalisme socialiste», qui dominait jusqu'à une date récente le champ russo-soviétique.

Cette filiation organique jette une nouvelle lumière sur le cadre morphologique général à l'intérieur duquel s'est développé le projet formaliste russe. Ce modèle explicite en particulier certains accents conceptuels des formalistes, à savoir les questions de l'approche immanente des phénomènes linguistiques et littéraires, de la diachronie systémique dans l'analyse linguistique et poétique, de l'évolution du système linguistique et littéraire et de la place du modèle téléologique dans ces théories. Cela permet de comprendre un ensemble de concepts formalistes et de situer epistémologiquement le phénomène du formalisme russe.

La conception organique surgit en Allemagne comme une tension entre l'optique des Lumières, qui privilégiait la recherche dans le domaine des langues aux fins de connaître les lois de la nature et de l'esprit humain, et l'optique du premier romantisme, qui souligne l'intérêt de la création populaire spontanée et de la puissance créatrice intuitive des peuples primitifs. Les investigations sur les antiquités et les valeurs nationales et les manifestations du Geist germanique des frères Schlegel et de Bopp en sont un aspect. Or, les recherches de dialectologie du russe et d'études folkloriques conduites par le premier Jakobson et par Pjotr Bogatyrev, dans le cadre du Cercle dialectologique de Moscou, ressemblent à ce phénomène. La grammaire comparée de W. von Humboldt se tourne vers la découverte du passé national. Or, le futurisme russe manifeste curieusement la même tendance en s'occupant de l'art populaire archaïque (il differe $\mathrm{du}$ futurisme italien sur ce point). Le futurisme du premier Jakobson cohabite parfaitement avec sa passion pour les antiquités folkloriques.

Le formalisme russe s'inscrit dans les luttes épistémiques entre les téléologistes et les mécanicistes qui ont marqué la fin du XIX ${ }^{e}$ siècle. Il fait la synthèse, d'une part, entre une vision téléologique, la fin prédéterminée propre du langage et de la littérature, et, d'autre part, une vision mécaniciste, selon laquelle l'univers langagier et textuel fonctionne comme un mécanisme en accord avec des lois naturelles intrinsèques. La genèse du formalisme montre une évolution vers l'explication synthétique du phénomène littéraire, où l'aspect téléologique (le Tout, la totalité, la fin prédéterminée) n'est plus en conflit avec l'explication strictement «matérielle», analogue à celle de la physico-chimie. Ce parcours se manifeste dans l'enchaînement successif des concepts de langage transmental (zaum'), de défamiliarisation (ostranenie), de procédé 
(priem), de dominante ${ }^{2}$, de construction, de visée (oustanov$k a$ ), de "structure subliminale», pour aboutir finalement au "phonème», lequel est défini dans toutes les langues du monde comme un faisceau de traits distinctifs. Une même vision universaliste se cristallise, durant les années 1920, avec les écrits du cercle de Bakhtine (M. Bakhtine, V. Volochinov, P. Medvedev), dans des concepts tels que «signe idéologique», "évaluation sociale», "pont idéologique», "idéologème», «dialogisme», «roman polyphonique», "carnaval». Bien des années plus tard, la même optique reparaît dans la sémiotique de la culture élaborée par l'École sémiotique de Moscou et de Tartu (I. Lotman, V.V. Ivanov, V. Toporov, etc.). Elle met en circulation les notions de programme, de modèle signifiant secondaire, de code, de traduction, de "structures extra-textuelles» fusionnées dans la totalité signifiante de la "sémiosphère». L'aspect téléonomique implique que tout le processus du développement individuel (ontogénèse) ainsi que tous les comportements des individus poursuivant un objectif sont guidés par le programme, et qu'ils sont réglés par l'existence d'un point final ou d'une règle de comportement.

Les romantiques allemands considéraient la langue comme un organisme vivant, où la définition linguistique de la structure, comme système de relations ordonnant un ensemble d'éléments, se mélangeait avec son acceptation biologique dans le domaine des sciences naturelles. D'où le rôle du sanscrit dans ces conceptions, qui visaient à percevoir à travers sa «morphologie transparente» un "état premier» du langage, à atteindre les «mots originaires». Si les romantiques comptent percevoir dans les racines monosyllabiques isolées le rapport signifiant qui va du son pur au sens, c'est qu'ils croient que les premiers sons doivent être signifiants pour eux-mêmes, et non en vertu de l'arbitraire du signe.

On trouve le même projet archéologique du côté des futuristes russes: Velemir Khlebnikov rêve de trouver la "pierre philosophale» permettant de fondre toutes les racines des langues slaves. Alexeï Kroutchenykh invente la «langue transmentale» universelle et compréhensible par tous. Viktor Chklovski essaie de dégager les "procédés purs» qui seraient au fondement de l'art. Roman Jakobson conceptualise le phonème, élément de base du langage, en réalisant le rêve futuriste d'accéder aux "cordes invisibles» des alphabets. Le cercle de Bakhtine insiste lui aussi sur la matérialité du signe et des manifestations sémiotiques de la conscience. S'inspirant du fonctionnalisme neuro-sémiotique du dernier Jakobson, la sémiotique de Iouri Lotman et de Vjatcheslav Vsevolodi- vitch Ivanov, élaborée dans les années 1960-1980, ancre les foyers de l'activité langagière et symbolique dans les tissus mêmes des structures organiques. La conceptualisation du binarisme fonctionnel des hémisphères droit et gauche, la matérialité signifiante du literaturnyï byt (de la «vie littéraire» ou du «quotidien littéraire»), lequel s'exprime par les objetssignes, prolongations organiques des codes des états successifs de la culture, sont autant d'exemples de cette dominante organiciste. Le structuralisme russe (l'École de Moscou et de Tartu) avance une optique anthropomorphique analogue dans l'étude du texte et des systèmes signifiants: l'homme est défini comme un ensemble de textes. Les métaphores organiques constitutives de la doctrine sémiotique de Lotman complètent le tableau. De même que Goethe lit, à partir de l'os intermaxillaire qu'il découvre dans le squelette humain, non pas la «lettre morte» de l'appartenance à une filiation animale, mais la totalité vivante, la puissance du langage en tant que faculté fondamentale de l'être humain, de même Lotman souligne l'importance de la lecture de la totalité signifiante par l'enracinement physiologique des dispositifs sémiotiques et par l'anthropologisation du "texte de la culture».

\section{Quelques circonstances de la naissance de la modernité littéraire}

La filiation entre la pensée du premier romantisme russe et le formalisme russe est assez controversée. En Russie, cette filiation semble être ignorée, et en Allemagne elle est loin d'être acquise. En France, l'idée de cette filiation (qui ne paraît pas avoir été commentée par les germanistes) a été lancée depuis le camp des poéticiens ${ }^{3}$, puis confirmée par le camp des philosophes. L'importante anthologie des textes romantiques, L'Absolu Littéraire. Théorie de la littérature du romantisme allemand, publiée en 1978 par Philippe LacoueLabarthe et Jean-Luc Nancy, renforce cette prise de position. Les affirmations des poéticiens reçoivent un fondement philosophique fondamental pour le contexte français et de même le «droit de cité» dans le monde intellectuel. Le premier romantisme allemand et les développements exposés dans l'Athenäum - concepts de critique, de genre, de poésie, de symphilosophie - sont perçus désormais comme ouverture dans la modernité critique ${ }^{4}$.

Le découverte tardive par les universitaires français du texte important de Walter Benjamin, Der Begriff der Kunstkritik in der deutschen Romantik (ce texte de 1920 a été traduit en 1986 sous le titre Le Concept de critique esthétique dans le romantisme allemand, par Lacoue-Labarthe et Lang), 
devient un point de départ de la réception «modernisée» de la doctrine romantique 5 .

Jean-Marie Schaeffer analyse la théorie du roman chez Bakhtine à partir de la théorie romantique du «Roman»: la filiation romantisme-formalisme semble être confirmée sur le mode négatif par le biais de la critique du formalisme par Medvedev/Bakhtine (Schaeffer, 1983). Pour sa part, Dominique Combe a exposé, dans Théories des genres, l'influence de la poétique et de la linguistique romantiques sur la pensée de l'Opoïaz (Société pour l'étude de la langue poétique), en particulier de Roman Jakobson. Il parle de l'historicité des genres littéraires prônée par le formalisme comme de l'héritage du romantisme allemand (A. Hölderlin et F. von Schlegel, Hegel) (Combe, 1992: 115). Laurent van Eynde parle de l'expérience romantique dans le domaine de la littérature en termes de "crise de la modernité» et y voit «la restauration de la vie» (Eynde, 1997: 20). Sa lecture des écrits théoriques essaie d'analyser le romantisme d'Iéna en tant que "courant représentatif d'un certain mode d'exister de l'humain", et comme "explication d'un certain mode d'investissement de l'homme dans la parole poétique». Eynde s'oppose aux «interprétations idéologiques» qui réduisent souvent le romantisme à "une géniale anticipation des théories de la différence ou de la dissémination» et qui s'interdisent «l'accès à la signification anthropologique de la création romantique» (ibid.: 201).

En Russie, il n'existe pas d'analyse de la poétique du premier romantisme, à l'exception notable du livre de Viktor Zirmundskij (1996), "compagnon de route» des formalistes de 1914, dont il sera question plus loin.

Jurij Striedter s'arrête longuement sur la contribution «morphologique» allemande à la théorie du formalisme russe pour souligner, en dépit des affinités superficielles existantes, des différences fondamentales. Dans ces deux cas, l'analyse est concentrée sur la forme; la différence des approches relève de deux critères, le langage et l'évolution. Si les théoriciens allemands, en accord avec les genres traditionnels, divisent la littérature en poésies lyrique, épique, dramatique, le formalisme russe, en revanche, distingue la poésie (vers ou langage poétique) et la prose. Du côté allemand, ces distinctions génériques sont établies en fonction des conceptions de Goethe, qui considère les poésies lyrique, épique et dramatique comme des "formes naturelles» et les associe à l'idée de la croissance organique de la littérature et de ses formes. Ces conceptions organiques, de même que les pures typologies, sont également étrangères aux théories formalistes (Striedter, 1989: 52-53).
En effet, le formalisme considère que le défaut essentiel du positivisme, dit aussi analytisme et atomisme, est de poser des «typologies pures»; il réside dans son incapacité d'embrasser le tout et de proposer un ensemble cohérent de l'évolution littéraire. Quant aux conceptions organiques et à la «croissance organique» des formes littéraires, cette optique reste virtuellement intégrée dans les théories formelles, qui sont entièrement pensées contre la "croissance organique» au nom de la "croissance non organique», procédant par sauts et «en ligne brisée». En d'autres termes, contre le modèle évolutionniste de la tradition organique et morphologique à l'allemande, le formalisme propose un modèle essentiellement mutationniste.

Selon J. Striedter, il ne faut pas surestimer les analogies entre les approches formelle et morphologique. L'intérêt pour la "forme artistique de l'œuvre poétique», que le formalisme partage avec les théories morphologiques, celle d'Oskar Walzel par exemple, et la dichotomie entre le contenu et la forme de l'œuvre poétique proposée par ce dernier épuisent la ressemblance. Walzel s'occupe non pas des traits spécifiques de l'art verbal à l'opposé des structures du langage «non littéraire», mais de l'«illumination réciproque» de tous les arts. À ce propos, la traduction d'un ouvrage de Walzel par Viktor Zirmundskij a affirmé sa rupture avec le cercle formaliste (Striedter, 1989: 53-54).

Néanmoins, les relations entre l'approche théorique symboliste et morphologiste, d'une part, et l'approche formaliste, d'autre part, sont loin d'être simples et univoques. A. Hansen-Löve souligne en effet que la théorie et la pratique du langage zaum' du cubo-futurisme entretiennent des rapports de dépendance complexe avec le principe de l'autonomie des formes artistiques mis de l'avant par la poétique symboliste. En effet, malgré le fait reconnu de l'influence des théoriciens symbolistes sur les "praticiens» futuristes et les «théoriciens» formalistes, dont les premières constructions sont expressément anti-symbolistes et visent à réfuter la «forme interne du mot» de leurs précurseurs au nom de la «forme externe», l'étude de la dépendance du «mot autotressé» de la poétique et, par conséquent, de la philosophie symboliste, rencontre deux difficultés: 1) la dénégation de cette liaison par les futuristes (et les formalistes eux-mêmes) et 2) l'absence de toute définition explicite de ce phénomène dans la poétique symboliste (Hansen-Löve, 1978: 99).

Ewa M. Thompson conclut son analyse comparée du formalisme russe et du New Criticism américain en affirmant que le formalisme représente une tendance "néo-positiviste», 
à la différence du «nouveau criticisme» américain, qui relève d'un courant idéaliste (Thompson, 1971: 149). Cet auteur voit le parcours formaliste comme une sorte d' "effacement" de ses sources «idéalistes» (le kantisme dans la version symboliste d'Andreï Biélyj) pour aboutir à une méthode scientifique dite «néo-positiviste». À partir de l'alliance des croyances positivistes du XIXe siècle et des théories idéalistes et symbolistes, les formalistes évolueraient vers une "scientificité» totale dans sa version du "positivisme logique» du XXe siècle (Thompson, 1971: 108-109). Cette interprétation commode est sans doute trop schématisante et réductrice par rapport à ce processus.

Partant de cette idée que le rôle et les effets du modèle organique dans la culture et dans l'histoire intellectuelle russe n’ont pas été encore suffisamment explicités et analysés, nous examinerons ici les "affinités électives» qui relient le modèle organique de l' «absolu littéraire» du romantisme allemand aux concepts sémiotico-littéraires du formalisme russe et du structuralisme.

Si nous souscrivons à la remarque de Victor Erlich sur l'impossibilité de simplement considérer le formalisme russe comme une version déguisée de la doctrine de l' «art pour l'art» de la fin du XIX ${ }^{\mathrm{e}}$ siècle, nous éprouvons en revanche une réticence à l'égard de sa définition de la démarche formaliste comme "néo-positiviste», et de sa thèse selon laquelle le formalisme russe n'a pas été concerné par l'essence ou par les buts de l'œuvre d'art (Erlich, 1955: 171). Nous considérons au contraire que le formalisme russe fait partie de la filiation organiciste ${ }^{6}$. Les développements suivants, qui visent à restituer les projets théoriques formaliste et romantique, doivent nous permettre de formuler les points de convergence entre les poétiques de ces deux courants.

\section{Le festin pendant la peste ou la dictature du concept}

Que reste-t-il aujourd'hui du formalismerusse? Le structuralisme et les sémiotiques sont-ils la continuation de l'Opoïaz? S'agissant des rapports entre les doctrines formaliste et structuraliste, il serait erroné d'y voir des relations de simple succession ou de filiation paisible. Le structuralisme et la sémiotique contemporaine se constituent en grande partie à partir du rejet de certaines conceptualisations formalistes: les deux projets semblent être différents.

Force est de constater que le structuralisme, pour qui la structure est le contenu logiquement organisé, élimine la notion de "forme», en tant que catégorie pertinente, et neutralise la notion formaliste de «matériau» par la notion de «struc- ture». Le structuralisme, qui n'a pas maîtrisé la distinction formaliste du matériau et du procédé, a beaucoup moins étudié la structure spécifique de la littérature. Les méthodes structuraliste et sémiotique n'assimilent pas les termes formalistes d'évolution littéraire, elles ne peuvent pas décrire la transformation des systèmes de genres ni reprendre le projet formaliste de construire la science de la littérature. L'opposition structuraliste «code-message» ne recouvre pas l'antithèse formaliste "matériau-procédé»/priem/. La notion formaliste de matériau n'est pas accessible à l'analyse structurelle et sémiotique. La notion formaliste de motivation ne peut pas non plus être traduite dans le langage du structuralisme.

Les structuralistes considèrent le texte essentiellement du point de vue de la sémantisation des éléments formels, tandis que les formalistes le font du point de vue de la formalisation du sémantique. Le formalisme comprend le contenu comme une forme et cherche le "caractère formé» (oformlennost) du contenu. Le structuralisme, au contraire, comprend la forme comme contenu et cherche le "caractère de contenu" (soderjatelnost) de la forme; le formalisme suit le cheminement du sens au signe, et le structuralisme va du signe au sens. Le formalisme part de la présomption du tout, de la totalité, tandis que le structuralisme part de la présomption de la partie.

Le structuralisme s'est occupé de la traduction en sa langue des vieilles notions formalistes, en croyant que le fait même de les avoir rebaptisées et de les avoir fait entrer dans un unique système terminologique réglait toutes les difficultés. Le "transcodage» infini d'une langue en une autre l'a mené à la création d'un domaine du savoir prétendument nouveau, fondé dans ce nouveau métalangage de traduction. Le structuralisme a ainsi créé un nouveau métalangage et non un nouveau domaine du savoir ${ }^{7}$. Ainsi, la spécificité de l'objet littéraire et la mise en relief du rôle morphogène de la substance sur la forme de l'expression préconisées par les formalistes, de même que la tendance formaliste à considérer la substance de l'expression comme instance formatrice de la forme artistique, se trouvent-elles dissoutes dans le cadre de l'approche structuraliste.

Au commencement de ce «roman familial», paraît en 1914 la «nouvelle» de la résurrection du mot. C'était peu après celle de la mort de Dieu et à la veille de la Grande Guerre. L'inertie, les automatismes ont «mangé» ces deux événements. Vivre avec le mot ressuscité s'est révélé en revanche plus difficile. Il fallait comprendre que la forme est quelque chose qui doit être vécu, que vivre signifie s'attribuer des formes. La vie étant perçue comme ce qui se déve- 
loppe et s'exprime dans et par les formes artistiques, la forme et la vie ne sont plus dissociées. Ce formalisme mélange la perception des formes artistiques avec la perception de la vie ou du "monde externe».

C'est ce qu'a formulé Viktor Chklovski dans le texte fondateur La Résurrection du mot (1914). La vie est une forme vécue et non pas un mode d'emploi. Car dans le cas contraire, les mots deviennent des "signes algébriques» perçus en dehors de leur forme interne (image) et de leur forme externe (son). Le mot est mort quand il se transforme en marque d'équivalence.

Et pourtant la découverte du principe d'équivalence est comptée parmi les acquisitions fondamentales du formalisme. Elle consiste à dire que l'équivalence est porteuse de sens, qu'elle "fait sens». C'est l'équivalence qui démembre les unités sémantiques par sa puissance syntaxisante, qui formalise le sémantique et qui fait surgir les formes dans le domaine de sens, là où Saussure ne percevait que des nébulosités amorphes. En lisant ce premier ouvrage de Chklovski, on observe que, malgré l'apparence de révolte contre une poétique symboliste, il insiste sur une perception du mot opposée à celle qui s'accomplit par la «reconnaissance». En fait, la fameuse ostranenie (ou défamiliarisation, ou Verfremdung), issue de la notion de «mot ressuscité», met en valeur des procédés «symbolistes" par excellence, comme la forme externe (son) contre la forme interne (image). Le conflit entre le symbolisme et le formalisme se situe sur un terrain essentiellement symboliste. On assiste à l'articulation du paradoxe qui surgit dès la naissance de l'Opoïaz et qui a duré longtemps après sa mort historique.

La théorie de la "méthode formelle», que résume $B$. Eikhenbaum dans un article de 1925 recueilli par Todorov dans Théorie de la littérature, n'a pas inventé la poésie, mais elle a inventé une manière de parler du langage poétique. La zaum', ou "langage transmental» des futuristes russes, est apparentée au métalangage du formalisme. Dans le schéma classique des fonctions du langage formulé par Jakobson en 1958, une affinité singulière relie ainsi la fonction poétique à la fonction métalinguistique. Celles-ci se distinguent radicalement des autres fonctions par leur caractère "autotélique» et immanent: elles ne visent pas autrui, elles sont repliées sur elles-mêmes. Jakobson considère la fonction poétique et la fonction métalinguistique comme des fonctions fondamentales du langage. Le formalisme russe est luimême un phénomène poétique par excellence, la quintessence de cette littérarité ou de cette poéticité qu’il recherchait.
La Résurrection du mot pose "comme trait distinctif de la perception esthétique le principe de la sensation de la forme», rappelle Eikhenbaum, qui cite Chklovski: «la perception artistique est cette perception dans laquelle nous éprouvons la forme» (dans Todorov, 1966: 43). Cette perception est «un élément de l'art» et l'art «n'existe pas hors de la perception». La forme alors, à la différence des théories transcendantales du symbolisme, "n'est plus une enveloppe, mais une intégrité dynamique et concrète qui a un contenu en ellemême» (ibid.: 43-44).

C'est la notion de "procédé» qui sert aux formalistes à renouveler et à dynamiser la notion de forme. Partant de l'affirmation d'une spécificité de la littérature, la méthode formelle s'achemine vers la sémantique. C'est une pensée de l'équivalence: les séquences équivalentes peuvent posséder une charge de fait sémantique. Pour étudier «les traits spécifiques de l'art littéraire», le formalisme part de la différence fonctionnelle entre langue poétique et langue quotidienne. En ce sens, le formalisme russe a été dès ses débuts un fonctionnalisme.

Le formalisme s'était donné comme objectif d'abolir la perception reçue du terme "forme» opposé au "contenu», d'arracher la "forme» du «fond». Le nouveau sens du terme de forme est lié à la distinction forme/matériau propre au formalisme: "le matériau de l'art littéraire est hétérogène et comporte des significations différentes" (ibid.: 63). I. Tynianov, cité par Eikhenbaum, conclut, dans Le Problème de la langue poétique, que pour le formalisme, "la notion de "matériau" ne déborde pas les limites de la forme, le matériau est également formel»; il n'est pas à "confondre avec des éléments extérieurs à la construction». La forme, définie comme dynamique, implique que «l'unité de l'œuvre n'est pas une entité symétrique et close, mais une intégrité dynamique ayant son propre déroulement", dont les éléments sont liés "par un signe dynamique de corrélation et d'intégration" (ibid.: 63-64). La théorie formelle est fondée sur trois distinctions majeures: langage poétique contre langage quotidien, procédé contre matériau, sujet contre fable.

À partir de ces dernières, la méthode formelle introduit un déplacement de perspective d'une forme intransitive de l'esthétique classique (la logique interne de l'œuvre) vers une perspective fonctionnaliste, celle-ci étant comprise comme mode d'emploi concret de telle ou telle forme. Chez les formalistes, la notion de procédé précède la notion de fonction: le procédé est conçu comme une unité qui transgresse divers «matériaux» artistiques. L’unité de procédé forme cette con- 
nexion entre diverses formes et divers matériaux. Les procédés étant compris comme uniques et monovalents, la notion de fonctionnalité apparaît comme conséquence de ce fait.

Le procédé est une instance formatrice ou morphogène: il se donne comme «une forme particulière du discours ayant ses propres qualités linguistiques", conclut Eikhenbaum (ibid.: 74). Il s'ensuit que le procédé se manifeste par des marqueurs linguistiquement pertinents et donc matériels. On suppose que les procédés different selon le but de leur emploi. Cette distinction des objectifs, qui correspond à la différence des procédés, entraîne la différence du "matériau ", à savoir des «qualités linguistiques» ou de la «substance linguistique».

Ces marqueurs matériels sont réunis par les formalistes sous le nom de "matériau». Le matériau est conçu comme «motivation du procédé». La symétrie initiale de procédés regroupe diverses motivations, c'est-à-dire divers matériaux. Cette «substance linguistique» est donc ce qui correspond à une visée ou à une charge intentionnelle exprimées par tel ou tel procédé. L'idée de l'identité du procédé sur des matériaux différents et de la différenciation du procédé selon ses fonctions mène les formalistes «à la question de l'évolution des formes, c'est-à-dire aux problèmes de l'étude de l'histoire littéraire» (ibid.).

Cette redéfinition de la forme par le terme de procédé entraîne d'autres concepts importants de l'Opoïaz: ceux de construction et de dominante. Le sujet ou la construction sont motivés par le matériau. La "dominante constructive» détermine le matériau et, par conséquent, la motivation. On peut dire, en résumé, que le sujet ou la construction exercent une pression sélective sur le matériau en fonction de leur charge intentionnelle. Le matériau sélectionné par une telle pression forme une motivation des procédés (c'est-à-dire des sujets ou des constructions) en question.

La conquête de la prose suit la conceptualisation de la poésie. Le formalisme déplace l'étude du sujet du niveau sémantique ou thématique au niveau formel ou syntaxique. Il affirme «l'existence de procédés propres à la composition du sujet»; le sujet cesse d'être "la combinaison d'une série de motifs", il se trouve transféré "de la classe des éléments thématiques dans la classe des éléments d'élaboration" (ibid.: 48). Cette formalisation du thématique et du sémantique change de nouveau le statut de la forme. Pour les formalistes, " la notion de forme s'était confondue peu à peu avec la notion de littérature, avec la notion de fait littéraire». Le formalisme accomplit ainsi «le passage de la poétique théorique à l'histoire littéraire» (ibid.) qui résulte de l'évolution de la notion de forme. Perçue dans sa variabilité permanente, celleci a conditionné la perception de l'œuvre littéraire non comme un fait isolé, un fait en soi, mais en relation avec d'autres œuvres.

On comprend la logique d'une telle transformation. Selon les premières formules de l'Opoïaz, une forme isolée, perçue dans son autonomie ou son "intransitivité», doit être sensible. Mais sa sensibilité même serait impensable sans d'autres formes, des formes "usitées», qui se donnent en fait comme une condition de sensibilité de cette nouvelle forme. La question de l'évolution littéraire est perçue par les formalistes comme un problème «du mouvement et du changement de formes", comme leur "succession dialectique» (ibid. : 65, 70). Dans l'affirmation formaliste - «la nouvelle forme n'apparaît pas pour exprimer un contenu nouveau, mais pour remplacer l'ancienne forme» -, Eikhenbaum voit une conséquence de

[...] la nouvelle notion de forme [...] comprise comme le véritable fond se modifiant sans cesse en rapport avec les æuvres $d u$ passé [...] et en tenant compte de son sens concret et de son importance historique. (Ibid.: 65)

La formule du premier formalisme, "l'art est un procédé constructif", est remplacée par le projet de différencier dans chaque cas particulier la fonction concrète du procédé. Le travail «sur une matière concrète» de l'œuvre littéraire oblige le formalisme «à compliquer la notion de procédé» par celle de fonction et enfin à remplacer la notion de procédé par celle de «signification fonctionnelle» (ibid.: 66). C'est ainsi que l'étude de la littérature, initialement comprise "comme une série spécifique de phénomènes», se transforme en examen des "faits historiques concrets", dicté par «la nécessité de tenir compte des fonctions concrètes de tel ou tel procédé», à savoir "la différence entre l'œuvre littéraire prise comme un certain fait historique et sa libre interprétation du point de vue des exigences contemporaines» (ibid.: 67).

Ce tournant fonctionnaliste, qui coïncide avec une pression idéologique du régime soviétique, marque la fin des tentatives de conceptualiser «l'évolution littéraire en elle-même» à partir de l'établissement de lois immanentes. Vers la fin des années 1920 , le formalisme aboutit à la nécessité de considérer avec la série littéraire d'autres "séries culturelles» : la notion de "fait littéraire» de Iouri Tynianov et celle de "vie littéraire» d'Eikhenbaum introduisent dans le champ d'analyse des aspects socio-économiques de la création. Mais même 
cette version «hegelianisée» de l'histoire littéraire reste essentiellement formaliste: car elle est confinée au dynamisme diachronique des formes, elle se donne comme «évolution hors de la personnalité», comme "phénomène social original». Le dernier formalisme a en effet découvert l'histoire: mais surtout comme un espace opérationnel qui «offre ce que l'actualité ne peut pas nous offrir: l'achèvement du matériau» (ibid. : 71).

\section{Le premier romantisme allemand et le formalisme russe}

Cette alliance de la critique et de la science, ainsi que l'inscription du formalisme dans l'histoire de l'avant-garde et de la Révolution russe expliquent la formule de B. Eikhenbaum, selon laquelle «l'Opoïaz a réalisé le modèle même du travail collectif» (ibid.: 73). Un autre célèbre modèle de création collective est fourni par le projet de "Symphilosophie» ou de "Sympoésie» de la doctrine romantique allemande (1796-1801). Cette production, où chacun crée l'un contre l'autre, est fondée sur l'idée essentiellement romantique du synthétisme ou de la fusion des contraires, anéantissant le principe de contradiction dans une logique supérieure (Todorov, 1977: 211-225).

Le principe de l'unité poétique interne, posé par Friedrich Schlegel au cœur du poème, fait converger la diversité de ses parties dans cette position unique, dans l'Un de toute signification. Cette intégration esthétique des parties à un tout, qui représente plus que la simple somme de celles-ci, s'insère dans la tradition organiciste, pour laquelle l'organisme se définit comme un passage d'une forme à une structure par le biais fonctionnel de ses organes. Le romantisme mise sur le structuralisme dynamique d'Aristote, à l'encontre de l'interprétation classificatoire du classicisme (Behler, 1992: 7879). Si la poésie est, pour les romantiques, la réalisation la plus parfaite de l'œuvre d'art, c'est qu'en poésie «l'on peut le mieux réussir l'intégration la plus variée et la plus cohérente d'éléments divers» (ibid.: 80-85). Le premier romantisme exprime, par exemple, cette idée par "forme», "construction», "organisation", "ensemble», termes apparemment homologues aux concepts formalistes.

Essayons de restituer la logique de ce cheminement de la vision romantique de la poésie vers la logique formaliste et structuraliste. Pour F. Schlegel, porte-parole de la «nouvelle sensibilité romantique», cette force d'intégration qui caractérise la poésie est liée à la notion de choix et au caractère délibéré "propre à la création artistique». Objet illimité, la poésie est la création artificielle ou artistique par excellence, car le concept de forme artistique s'oppose par son caractère délibéré "à l'image de la croissance organique» (ibid.: 8485). La perfection dans la forme de la poésie par rapport aux autres arts réside dans le fait que la poésie n'a pas d'objet délimité qui lui soit propre. Cette absence de limitation distingue la totalité propre à la poésie de celle d'un être organique, qui se donne comme une «totalité close» et qui se définit par ses propres limites. Par conséquent, la poésie est une totalité non organique; sa force d'intégration se révèle inorganique ou artificielle. Forme artificielle ou secondaire par rapport aux formes de la langue (unanimement conçue par les romantiques comme un être organique), la poésie morcelle et redistribue les formes de langue existantes en leur attribuant un nouvel ordre maintenu par une tension constructive du poème.

La fonction métalinguistique de la poésie s'exprime par le fait qu'elle fournit des modèles relatifs au fonctionnement du système de langue qui schématisent son dispositif. La poésie, forme artificielle inorganique, réutilise, selon ses lois immanentes, les formes toutes faites de la langue, elle crée les formes au second degré. La poésie incarne, pour les romantiques, cette «composante moderne, artistique» de leur théorie littéraire: il s'agit de la réflexion et de l'autoréflexion (ibid. : 213). Dans l'esprit de la philosophie réflexive, la poésie, de même que le moi de Fichte ou l'Esprit de Hegel, se définit en se pensant elle-même. Elle est une pure pensée textuelle de soi. Devenu autoréflexif, le texte poétique se pense: de fait, on atteint déjà la notion d'écriture. Comme en philosophie, en poésie «la connaissance de la connaissance est devenue consciente». Le projet de la "poésie transcendantale» de F. Schlegel pose l'idée de la poésie en tant que «conscience artificielle, conscience de la conscience» et "pensée réfléchie dans l'art comme élément poétique essentiel» (ibid.: 213).

Pour Schlegel, l'essence de cette poésie vient de ce qu'elle présente "avec le produit, l'élément producteur» (le poète avec le poème), qu'elle crée par là «la réflexion artistique et le beau réfléchissement de soi». Cette poésie transcendantale est censée «dans chacune de ses présentations se présenter aussi elle-même, et être partout à la fois poésie et poésie de la poésie», le terme «transcendantal» désignant ici, au sens kantien, la forme de connaissance qui s'intéresse surtout à la façon de connaître les objets et qui lie indissolublement le sujet connaissant et l'objet de la connaissance (ibid. : 213-214).

Cette conception "essentiellement moderne» de la littérature, qui abolit les frontières entre la philosophie et la poésie, semble anticiper la notion de fonction poétique chez 
Jakobson, pour qui elle dépasse largement les limites génériques de la poésie et se donne comme une fonction fondamentale du langage. C'est que les deux notions sont profondément «romantiques», c'est-à-dire qu'elles associent la mimesis et la poiêsis et saisissent l'œuvre verbale dans son devenir. Pour Jakobson, la fonction poétique est conçue comme la mise à nu du dispositif langagier, et elle annonce la naissance de la phonologie. La poésie, auto-conscience ou réflexivité incarnée du système linguistique, reflète l'armature grammaticale et la charpente sonore du système, elle en fournit un "phénomène originaire» (Urphänomen). Ces deux visions sont romantiques par leur universalité et par l'omniprésence attribuée à la poésie.

L'œuvre poétique étant pour Novalis un pur réseau de relations entre les éléments constituants, elle offre, comme la linguistique et la logique, "une science supérieure des connexions» et, par conséquent, «l'algèbre est la poésie» (Todorov, 1977: 215). L'idéal "autotélique» de la fonction poétique s'exprime dans cette profonde affinité qui lui fait manifester seule la «merveilleuse nature» du langage lui-même.

Pour le romantisme allemand, le beau est situé dans l'acte de devenir et de création (K. Moritz, Sur l'imitation formatrice $d u$ beau, 1788). Cette thèse essentielle entraîne une panoplie de conceptsauxquels fait écho l'ensemble des conceptions formalistes. Dans les deux cas, la même optique transformiste détermine les approches romantique et formaliste des phénomènes littéraires, conditionne aussi leur vision organiciste fondée sur l'idée de mort et de régénération permanente des formes et sur l'idée des rapports fonctionnels entre le tout et ses parties, entre les touts monades dans le tout englobant de la monadologie. L'un et l'autre traitent l'œuvre d'art comme une totalité vivante, comme un être organique dont l'évolution, à l'instar de l'évolution biologique, se compose d'une série de récapitulations qui font resurgir l'ontogenèse dans la phylogenèse de l'organisme littéraire.

L'ensemble des concepts littéraires du romantisme allemand offre au formalisme russe son futur cadre de développement. La fameuse distinction de Schiller entre poésie «nouvelle» et «ancienne», entre poésie «sentimentale» et «naïve»; le concept de signes motivés, que F. Schlegel et Novalis postulent à l'origine de la langue, qui est pensée en tant que pure expression, donc motivée pour devenir ensuite seulement un ensemble d'unités arbitraires; la poésie perçue par Novalis comme "force extra-mécanique»; la distinction Gestalt/ Gehalt, forme organique ou interne contre forme mécanique ou externe, ainsi que la distinction entre «l'ancienne poésie» comme "plastique et architectonique» et la "poésie moderne» comme "pittoresque ou harmonieuse» faite par A. Schlegel; le langage comme activité (energea) et non comme ouvre (ergon) de W. von Humboldt, voilà autant d'aspects qui articulent cette tension fondamentale pour le formalisme entre le langage poétique et le langage de communication, clivage qui déclenche l'ensemble des concepts formalistes. Le substrat symboliste des conceptions allemandes est le pôle de répulsion du terme «le mot auto-tressé» (samovitö̈e slovo) des futuristes russes A. Kroutchenykh et V. Khlebnikov et repris par les formalistes, par contraste avec la notion de «forme interne du mot» (vnoutrenneja forma slova), fondamentale pour l'école fondée en Russie par le linguiste Aleksandr Potebnja (1835-1891), admirateur de Schelling et de W. von Humboldt. À l'opposé de la forme organique interne, donc indissociable, le formalisme pose la nécessité artistique de briser les formes pour accéder aux noyaux combinatoires de tout art.

Le langage poétique en tant qu' «indicible» (selon Novalis, aucune expression désignant un concept déterminé ne peut être trouvée pour l'idée esthétique); la poésie comme «idéal d'une relation musicale pure» (L.Tieck, E.T.A. Hoffmann); la poésie comme "infini déjoué» et «sens de l'illimité» (Jean Paul); le concept de Transzendentalpoesie et d' «arabesque» comme "peinture fantastique absolue» (F. Schlegel) ont tous pour analogie la définition formaliste du «langage poétique» comme «langage transmental» (zaum). À l'opposé du catalyseur de diverses modifications de la «forme interne» à l'allemande, le langage poétique transmental est décrit par les formalistes comme langage désémantisé et devenu perceptible. La cohésion et la connexion des éléments poétiques («belle totalité» de l'œuvre d'art, selon l'expression de K. Moritz), la poésie comme «le deuxième monde singulier dans le monde d'ici» (Jean Paul) et la poésie comme «moyen de percevoir le tout» et comme "ordre supérieur» (F. Schlegel) correspondent au concept de dominante de R. Jakobson, ainsi qu'aux concepts formalistes de construction, de visée (oustanovka), de «caractère étroit de la série poétique» de Tynianov. Le "cercle herméneutique» de F. Ast et de F. Schleiermacher (avec la thèse majeure: toute la lecture critique est cyclique), ainsi que le concept de "poésie de la poésie» et de «réflexion poétique» (F. Schlegel) correspondent aux notions de parallélisme à distance et de «grammaire de la poésie» chez Jakobson. Le concept d' «ironie» et de «mot d'esprit excentrique» (arabesker Witz) de F. Schlegel 
correspond au concept de "parodie» de I. Tynianov. Le fameux concept d'ostranenie (défamiliarisation, ou Verfremdung pour Brecht) rappelle vivement les concepts de "Phantasie» (imagination) et d' "arbitraire sans entraves» (unbedingte Willkür) de F. Schlegel, ainsi que la définition du sens du courant romantique par W. von Humboldt comme «la vivacité du sens".

Synthétisme, symphilosophie et sympoésie, dont l'esprit traverse les fragments de l'Athenäum (synthétiser les individus en vue de la production d'êtres complets, rêve de la production et de la création collectives), expriment le rêve romantique de la production et de la créativité collective. La "poésie comme réalité» (F. Schlegel) et comme «réel absolu» (Novalis), ainsi que l'aspiration romantique à une nouvelle mythologie artistique (F. Schlegel), la mythologie de la raison «au service des idées» (Schelling), déterminent et délimitent la dimension politique de la pensée romantique qui mène au projet de l' «État organique» 8 . Dans le dernier formalisme et le «futurisme de gauche», cette tendance trouve un écho dans le concept de "commande sociale» (O. Brik) et dans son projet de se mettre au service de la révolution d'Octobre, qui aboutit au passage du formalisme au constructivisme. L'inspiration romantique de l' "absolu littéraire» fonde le projet formaliste d'extraire les procédés et les éléments de base pour briser les liens du quotidien et établir de nouveaux rapports entre les éléments atomistes afin de construire la nouvelle réalité.

Ainsi, les concepts du romantisme allemand constituentils le «noyau morphologique virtuel» des théories formalistes russes. Le «mot auto-tressé» et le «langage transmental» se donnent comme opposition constitutive au concept de «forme interne». Dès le premier texte de Chklovski de 1914, le formalisme ne démord pas de cette thèse qui trouvera sa formulation achevée dans la notion d' «ordre étroit de la ligne poétique» et du "trait sémantique fluctuant» de Tynianov, ainsi que dans la poétique des parallélismes chez Jakobson. C'est à partir de ce «noyau apophatique» que leur élaboration même est devenue possible. Tout se joue autour de la distinction entre la forme mécanique (ou la forme externe) et la forme organique (ou la forme interne). C'est par rapport à cette notion issue de la grande tradition de la formation (Bildung) que se structure le champ théorique russe. D'un côté, les théoriciens de la forme interne, de Potebnja aux poètes-théoriciens symbolistes, pour qui la forme externe en littérature n'est qu'une conséquence de la croissance interne à partir des germes qui renferment, dès le début, toute réalisation morphologique «de surface». De l'autre côté, le formalisme, réaction anti-symbolique, qui rejette la morphogenèse intrinsèque au nom de la forme externe, définie comme arbitraire ou "anti-organique». En troisième lieu, on peut citer le cercle de M. Bakhtine qui, tout en restant dans l'organicisme morphologique à l'allemande, modifie l'idéologie immanente de la forme organique, par l'apport dialogique ou polyphonique, et place le foyer de formation en dehors du texte littéraire, dans l'espace d'interaction sociale ou du «mot bivocal». La critique du formalisme par Medvedev et Bakhtine consiste à révéler sa contradiction théorique, à savoir qu'il ne reconnaît pas sa dépendance à l'égard de la philosophie romantique et qu'il essaie de réconcilier l'optique classique immanente et l'idéologie romantique transformationnelle en pratiquant «des descriptions «classiques» (aristotéliciennes) à partir de prémisses idéologiques romantiques" (Todorov, 1984: 86-87).

La divergence entre Saussure et Jakobson sur les rapports du synchronique et du diachronique dans la description linguistique (qu'E. Holenstein appelle «l'antithèse de Saussure et la synthèse de Jakobson» [1974: 35]) est méthodologiquement homologue à l'opposition entre l'optique organiciste de la morphologie allemande et l'optique analytique des Lumières. Jakobson se réfere à maintes reprises au concept d' "affinité» (en provenance de Wablverwandtschaften de Goethe), tantôt dans ses études phonologiques ${ }^{9}$, tantôt dans ses Dialogues avec K. Pomorska (Jakobson, 1980) ${ }^{10}$.

Comme la morphologie de Goethe, la linguistique et la poétique de Jakobson sont à la recherche d'un «type primitif» (Urtypus) d'un système donné. Le sens de la démarche morphologique consiste à percevoir à travers la diversité des formes de la vie «une seule forme principale» dont la modification produit cette panoplie (Wachsmuth, 1966; Lacoste, 1997). L'objet de la science de Jakobson - l'invariance dans la multiplicité et l'invariance des relations entre le tout et les parties - est un objet essentiellement «morphologique». Son projet phonologique et poétique part de l'idée d'un fond immuable sur lequel apparaît toute donnée de sensation; ce fond garantit la cohésion du tout dans les variations du système. L'influence du tout sur ses parties fonde la science de Jakobson, tantôt dans son aspect dynamique (la dépendance de toute donnée du champ), tantôt dans son aspect statique (le tout est plus que la somme de ses parties) (Holenstein, 1974: 24-25). L'invariance linguistique reçoit chez Jakobson le statut de fait symbolique, car elle se trouve valable au niveau non seulement intralingual (à l'intérieur de chaque langue), 
mais aussi au niveau interlingual (entre différentes langues). Sa science établit les «relations primitives» ou les "éléments primitifs», les «Ur-relations» ou les «Ur-éléments», qui précèdent tout système linguistique concret et qui se donnent par conséquent comme inter-systémiques. De même, le phonème de Jakobson, défini en traits distinctifs, dépasse la dimension d'un seul système fermé, dont la clôture seule est censée lui attribuer son caractère opérationnel. La dimension ainsi introduite transcende l'ensemble des systèmes linguistiques, définis comme arbitraires et fermés, et relève par conséquent du symbolisme universel.

Jakobson se concentre sur l'étude des relations intérieures et extérieures dans une perspective convergente. Son unique objet, l'équilibre dynamique, expression de l'économie générale intrinsèque au système, laisse constamment percevoir le Urtypus goethéen. Cet équilibre dynamique s'établit par le jeu réciproque des axes de convergence et de divergence, en fonction du principe général de complémentarité. Le schéma fonctionnel du langage (Jakobson, 1963: 220), qui pose l'autonomie des fonctions en tant que sous-codes dans le code général de la langue, illustre le dispositif des interdépendances des moyens linguistiques et de la perception qui fondent l' «économie interne» du système. En réinsérant les changements phonétiques dans l'ensemble du système phonémique qui subit cette mutation, Jakobson voit la téléologie du système dans sa visée de conserver l'ordre interne par un cycle de changements phoniques dirigé vers la stabilité initiale. Cette «Ur-relation», relation phonémique originaire, apparaît comme une unité primordiale; elle garde son statut immuable à travers toutes les transpositions phoniques. Ce noyau virtuel stable apparaît comme le but des modifications du système, il est opposé au matériau plastique des métamorphoses et acquiert le sens par cette invariance réitérée. L' «unité homonome» (ibid.: 39), qui réunit chez Jakobson l'axe synchronique et l'axe diachronique (qui sont antinomiques chez Saussure), affirme la visée téléologique de ces axes vers la relation originaire, qui se donne par conséquent comme le condensé de la philosophie du Urtypus. Méthodologiquement, Goethe, avec sa Urpflanze, et Jakobson, avec son projet phonologique intégral, traitent toute modification «en fonction du système à l'intérieur duquel elle a lieu» (ibid.: 45). Pour le poète et le linguiste, "la forme la plus évidente» d'une modification intrasystémique est «le rétablissement d'un équilibre à l'intérieur d'un système» (ibid.) qui vise à recréer la symétrie de départ (Ursymetrie). Le mouvement du système se trouve éclairci par le principe initial d'un équilibre interne: les tendances au rétablissement agissent comme la conséquence de la destruction préalable de l' "harmonie préexistante».

Mais c'est surtout dans sa poétique qu'apparaît «une secrète parenté» (Jakobson, 1977: 30), définie parfois comme «un élément sui generis [...] que l'on ne peut réduire mécaniquement à d'autres éléments» (ibid.: 45), ou comme "poéticité»:

[...] en général, la poéticité n'est qu'une composante d'une structure complexe, mais une composante qui transforme nécessairement les autres éléments et détermine avec eux le comportement de l'ensemble. (Ibid.: 46)

Cette "poéticité», qui vient remplacer chez le Jakobson de Prague le terme de "littérarité» de sa période formaliste russe, semble avoir un "parent secret» inattendu. Ce terme délibérément "anti-mécaniciste» et "anti-arbitraire», à fort "potentiel pénétrant», établit un curieux rapprochement avec le rêve galvanique de Novalis. Novalis recourt à cet «esprit galvanique» qui rend possible les "transitions» entre l'organique et l'inorganique, à ce fluide (dit aussi «amour et volupté») dans le langage humain, et qui incarne la "plasticité originelle», «inépuisable, sans limites», pour définir la poésie. Le poète conçoit ce fluide, "instance vitale», "force nourricière des solides", à l'instar des formulations de Ritter et de Baader, où cet élément circule entre les corps solides et les nourrit en constituant la «médiation galvanisante» dans la nature (Ayrault, 1976: 131-152).

Cette «troisième force» énigmatique, qui intervient dans l'action des deux forces fondamentales (attraction et répulsion), engendre les "affinités organiques» entre des systèmes génétiquement hétérogènes. C'est dans cet espace des affinités que le poète rencontre le poéticien, chez qui la fonction poétique est conçue comme instance transformatrice et animatrice du système de langage.

\section{Le "mécanisme primitif» chez les formalistes}

Le point de départ du formalisme est l'idée de la noncoïncidence productive des relations entre la forme et le contenu. Ce clivage constitue le moteur de l'évolution du système. Le nouveau phénomène dépasse dans son dynamisme les formes achevées existantes que lui fournit le système. La nouvelle forme, élément révolutionnaire, dépasse le contenu, élément conservateur, elle se crée un nouveau contenu.

La triomphe de la «branche cadette», la loi de la «canonisation» des genres vulgaires ou de la périphérie du système 
aux dépens de son centre, révèle le mécanisme fondateur de cette pensée. C'est le modèle descendant, le modèle du système en dégradation ${ }^{11}$. La métaphore d'une "marche du cavalier", qui illustre pour Chklovski la transmission de l'héritage littéraire, reprend en outre l'idée d'une mutation, à l'intérieur du système, conçue à l'instar de la mutation biologique. Les conceptions de Tynianov reflètent la même vision: la visée (oustanovka), en tant que moteur de l'évolution du système, agit comme un type de déformation imposée à la structure; déformation qui mutile l'ensemble structurel en fonction d'une dominante à l'œuvre. Une référence nettement biologique apparait dans ses carnets: il note en particulier l'idée du «sujet littéraire» comme un "gène en mutation".

La vision formaliste, qui conditionne a priori les résultats de leurs recherches (malgré de multiples affirmations du contraire), est un système d'enchevêtrements où tous les composants sont nécessairement présents dès le début, assurant ainsi l'intégralité, la fonctionnalité et l'existence même du système. Le dispositif de la dominante assure la «mise en valeur » d'un seul élément, qui aboutit à la transformation de tous les autres, sans pour autant éliminer ces autres éléments qui restent toujours présents dans le système. Il s'agit d'une permutation dans l'ensemble systémique, processus qui témoigne de l'extrême flexibilité et vitalitéde cet ensemble. Cette permutation apparaît comme un "phénomène primitif» (Urtypus), où l'intégralité du système est conditionnée par la possibilité constante du retour au point de départ. L'équilibre interne du système reste stable malgré les transformations, il perce à travers les modifications possibles et se donne comme le fondement de l'identification du système.

La métamorphose est perçue par les formalistes comme un mécanisme essentiel de l'évolution littéraire. Dans l'optique de la méthode formelle, cette métamorphose est présentée en termes de transformation des tissus organiques, c'està-dire en termes parfaitement biologiques. L'élément de départ est perçu comme un élément en devenir, c'est-à-dire non pas comme une instance déterminée, fixe, produit du devenir, mais comme un élément instable et sensible aux facteurs externes. Il se transforme sous l'influence de facteurs externes (mais toujours «systémiques») et, finalement, il montre une flexibilité extrême qui peut aller jusqu'à une transformation complète de l'instance de départ.

C'est ainsi, par exemple, que Tynianov présente la transformation du genre de l'ode: l'ode, le genre «aîné», existe non pas comme une unité fermée et autosuffisante, mais comme "une certaine visée constructive» (izvestnoje kons- truktivnoje napravlenije). Les autres formes lyriques ne perturbent pas l'existence de l'ode car elles sont perçues comme «cadettes». C'est pourquoi le "genre élevé» de l'ode pouvait attirer et «absorber» toutes sortes de matériaux nouveaux, l'ode "pouvait se réanimer aux dépens d'autres genres», elle "pouvait enfin se transformer jusqu'à un degré méconnaissable»; néanmoins, elle ne cessait pas d'être perçue comme une ode tant que les «éléments formels restaient attachés par la visée (ustanovka) à une fonction discursive essentielle» (Texte der russischen Formalisten, 1972: 316, c'est moi qui traduis). Cette "visée», incluse dans la nature même du genre, subordonne et transfigure tous les éléments du mot (ibid.: 308). Le mécanisme de cette évolution s'accomplit donc par la mutation, c'est un dispositif essentiellement mutationnel.

Par conséquent, le processus de l'évolution littéraire est situé dans la conscience du récepteur. La conscience de la valeur du genre se matérialise par l'attachement des éléments formels à une fonction discursive précise: cet attachement apparaît sous la forme de visée. La forme est une construction volontariste qui incarne une certaine valeur appartenant à la conscience du récepteur ${ }^{12}$.

Le poème présente le dualisme des séries et leur corrélation imminente. Pour Ossip Brik, le phénomène du vers apparaît comme une tension entre deux principes constructifs - qui jouent le rôle de deux pôles opposés, ou de deux forces fondamentales, entre lesquels s'accomplit la réalisation d'un phénomène. Pour le vers, les deux limites constitutives deviennent le mot sémantique, en provenance du discours quotidien, et le mot «transmental» désémantisé, perçu comme la réalisation sonore d'une certaine exigence rythmique.

Le vers est donc une tension entre le principe sémantique abstrait (orientation au sens caractéristique du discours en général) et le principe rythmique abstrait (orientation au rythme caractéristique uniquement du langage poétique). L'exigence sémantique par rapport au vers apparaît donc dans une situation où les exigences rythmiques deviennent trop saillantes et «menacent de transformer le vers en langage transmental». L'exigence de renforcer les instances du langage «normal» quotidien dans la parole poétique est, selon Brik, une réponse à cette rupture entre les séries rythmique et sémantique (ibid.: 184). La conclusion de Brik est la suivante: Il en découle que dans tous les temps et à toutes les époques il a existé deux types d'attitude à l'égard du vers: l'accent mis sur l'aspect rythmique et l'accent mis sur l'aspect sémantique. Cette contradiction se renforce surtout pendant les périodes critiques de la culture poétique. (Ibid.) 
Du point de vue formel, l'un de ces deux principes est dominant dans les cultures poétiques de diverses périodes. L'évolution du vers se réalise par la lutte contre le type poétique dominant.

Cette optique rappelle immédiatement le principe organique formulé par Goethe - son idée de balance ou de «budget de la nature", de l'économie intrinsèque du système organique clos, de l'équilibre interne de l'organisme ${ }^{13}$. La même idée de balance ou d'équilibre intrasystémique fonde l'approche de la "poétique moderne", que Brik formule contre l'opinion du "bon sens", qui considère les procédés poétiques comme un supplément décoratif (ornement) ajouté à l'ordre normatif de parole. Cette approche «moderne» considère comme fondement de la parole poétique ce qui est naïvement perçu comme un ornement. Et au contraire, la valeur sémantique du complexe rythmique est, sinon un ornement, alors une concession nécessaire à l'égard de la conscience non poétique. «Si tous les hommes pouvaient penser avec des images transmentales, aucune élaboration sémantique de la parole poétique ne serait nécessaire» (ibid.: 210).

Les «nouvelles unités» introduites par Brik dans l'analyse du poème présentent à l'état pur la «loi morphologique» de la construction poétique. Cette dernière, telle la loi de la polarité, est composée de deux facteurs: dans la ligne, unité poétique fondamentale, les mots se trouvent combinés selon une loi rythmique précise et, simultanément, selon les lois de la syntaxe prosaïque (ibid.: 190-192). La loi constructive du texte poétique formulée par Brik esquisse la loi générale de tout «système signifiant secondaire»: le principe d'équilibre dynamique, du mécanisme compensatoire à l'œuvre dans le texte "artistique».

Un autre formaliste, Sergueï Bernstein, considère l'œuvre d'art comme un système expressif sui generis, comme un signe externe du système émotionnel dynamique des éléments non sensibles qui se réduisent aux "émotions abstraites" ${ }^{14}$. Le contenu artistique est introduit par l'intermédiaire d'un signe externe qui constitue un objet esthétique, objet de la perception et de l'évaluation esthétique. Ce contenu se crée dans la perception sur la base de ce signe. Bernstein perçoit cette synthèse du contenu comme un fonctionnement de l'œuvre d'art en tant que signe, fonctionnement conditionné par sa structure (ibid.: 342). L'œuvre d'art, ainsi que la forme au sens large, se trouve donc définie comme une donnée sensible qui sert de signe à ce contenu. Bernstein souligne que l'œuvre d'art se donne comme totalité, dans la mesure où celle-ci n'accepte pas un démembrement en parties ou en éléments. Les phénomènes matériels qui correspondent à un système de notion esthétique ne peuvent être séparés les uns des autres comme des éléments qui constituent cette totalité esthétique. Dans l'œuvre d'art, on a affaire non pas aux éléments mais aux facteurs, et chaque parcelle de matière qui y est utilisée concentre l'interaction de ces facteurs. Pour Bernstein, du fait que les facteurs de l'ouvre d'art ne puissent pas être dégagés par le démembrement mécanique, le seul moyen de les établir se réduit à la définition des points de vue acceptés ou plutôt provoqués par un objet donné. La structure de l'objet est définie par l'ensemble des points de vue exigés par cet objet (ibid.: 344).

Cette appréhension dynamique du «matériau verbal», de la «substance de l'expression» d'une construction artistique, mène à définir le «mot» comme une construction vide en soi, doué d'une plasticité illimitée et, par conséquent, d'un potentiel morphologique puissant. En d'autres termes, l'unité minimale de la construction poétique, sa «brique» matérielle, est un équivalent d'un "phénomène primitif» qui présente à l'état condensé et replié toute la panoplie des développements possibles ${ }^{15}$. Ainsi défini, le mot rejoint l'ordre des "phénomènes originaires» - «le roman le plus typique de la littérature universelle», une "nouvelle», un "conte» ou un «sujet" primitifs -, phénomènes qui ne sont que des effets de croisements de séries, pures manifestations de lois polaires, du dualisme interne du système. C'est une unité morphogène en puissance, qui contient virtuellement toute la richesse morphologique à venir.

\section{Conclusion}

La possibilité de poser la filiation entre la pensée romantique allemande et le formalisme russe se fonde sur les points de convergence suivants:

1. L'abandon de la tradition mimétique dans la théorie de la littérature et la redéfinition de la mimesis comme poiêsis, donc le postulat de la mimesis poüetique.

2. L'immanence du domaine littéraire et linguistique: postulat des lois internes qui gouvernent l'évolution du système de l'intérieur, c'est-à-dire sur le mode organique.

3. L'historicisation et la relativisation radicales des concepts relatifs à la théorie de la littérature: accentuation du système en devenir, accentuation des traits dynamiques, c'est-à-dire historiquement changeants des constructions théoriques; attachement des catégories de la théorie littéraire (distinctions génériques, procédés, thématique) aux moyens linguistiques de leur expression. 
4. Le remplacement sur le plan évolutionniste (diachronique) de la métaphore de l'organisme (romantisme) par l'idée du système (formalisme). Ces deux conceptions restent néanmoins fondées sur la présupposition de la pertinence de la dimension diachronique pour l'état synchronique du système et sur l'implication du téléologisme interne. On peut donc parler d'une ambiguïté organique inextricable, propre à ces deux visions, qui fait osciller les théorisations entre divers motifs de cette systématicité.

5. Le transfert de l'objet de recherche du niveau graphique (lettres, étude étymologique) au niveau sonore (son, phonème): le formalisme achève, pour la science de la littérature, le tournant romantique pour les sciences du langage, par le fait qu'il remplace l'analyse des signes matériels graphiques par l'idée de l'évolution des éléments (sons, procédés) au sein du système. En d'autres termes, il s'agit d'un passage du graphème au phonème, d'une Augenphilologie à une Ohrenphilologie.

6. Le modèle binaire ou polaire des constructions théoriques, dont les composants interagissent mutuellement en fonction du principe de "changement de ton" (Tonwechsel), de la transmutation des éléments (romantisme) ou de la dominante (formalisme, structuralisme). La complémentarité fondatrice des modèles théoriques: accentuation du fragment et du fragmentaire, perçus comme expression de la sensibilité «moderne» versus l'achevé et l'harmonieux propres au classicisme (romantisme); l'opposition rythmique-sémantique; ode-élégie; prose à sujet-prose sans sujet, etc. (formalisme).

7. Le nouveau concept de critique littéraire s'exprime comme un avènement de la «modernité critique». Il pose l'exigence d'une «auto-réflexivité» (Selbst-Reflekzion) du texte littéraire, comme introduction de la dimension «méta-poétique» dans l'œuvre littéraire. Cette figure d' «auto-reflexivité» met l'accent sur la nature «technique», "fabriquée», "arbitraire», à savoir corrélative ou «organique» de l'œuvre d'art. Ce redoublement de la production littéraire et du travail critique (prise de conscience) de ce texte par son auteur (lecteur) est devenu un trait distinctif de la situation actuelle des études littéraires. 8. La distanciation ou la «défamiliarisation" propre à l'œuvre d'art: la mise en valeur de l'ironie (romantisme) et de la parodie (formalisme) comme un procédé universel de la création artistique ainsi que de l'«évolution littéraire».

9. Le fait que les deux courants se fondent sur le modèle de l'évolution descendante: le système se développe par la décomposition de l'unité initiale et par le déclin, c'est-à-dire par la perte de la perfection primitive (romantisme) ou du syncrétisme préalable (formalisme, structuralisme).
10. L'idée de l'«hybridation productive»: le mélange des genres (romantisme), les branches ancienne et cadette en évolution littéraire, la dominante, la visée, l'évolution littéraire, la «vie littéraire» (formalisme), le dialogisme, le polyphonisme, le roman (Bakhtine et son cercle). L'interaction entre les systèmes autonomes est conçue, sous l'influence de modèles en provenance de la biologie, comme un moyen productif qui mène à l'apparition d'un nouveau système.

11. Le vaste recours des auteurs « romantiques» et des formalistes au discours scientifique en provenance du champ d'autres «sciences» (sciences exactes et sciences de la nature) et l'assimilation dans ses constructions théoriques des modèles d'autres branches épistémologiques.

12. Le modèle prospectif ou téléologique comme mode d'organisation et comme lien entre la pensée romantique et formaliste.

\section{Notes}

1. Le modèle organique issu de la tradition allemande de formation (Bildung) se manifeste avec force dans la morphologie, science de formes vivantes, fondée par Goethe dans ses écrits de philosophie naturelle. L'objet de la morphologie est la Gestalt (la structure), la formation et la transformation des corps organiques ou des totalités organiques vivantes. La morphologie se donne comme alternative aux sciences naturelles "classiques" (anatomie, physiologie) qui recherchent l'explication en termes de lois causales. Lubomir Dolezel résume ainsi les principes de la morphologie de Goethe:

1. La structure organique est une entité autosuffisante et complète formée par l'interrelation de toutes ses parties. La créature vivante est un univers en soi qui existe à cause de lui-même et par lui-même, il est un but en soi. Comme ses parties sont reliées entre elles par des interactions réciproques, l'animal accompli renouvelle constamment le cycle de la vie et il est à considérer comme physiologiquement parfait.

2. Le tout est plus que la somme de ses parties. La différence entre un ensemble non structuré et la totalité de l'organisme se manifeste dans la capacité de ce dernier d'acquérir des propriétés additionnelles, supplémentaires. La totalité structurelle de l'organisme assure sa vie; ni les parties individuelles ni leur ordre ne peuvent être changés sans qu'ait été détruite la structure. Si elle est perturbée, la totalité organique ne peut pas s'engendrer de nouveau à partir de ses restes.

3. La structure est une unité d'oppositions polaires. Pour Goethe, l'essence même de la nature se manifeste en oppositions binaires. La vie de la nature consiste à diviser l'unité et à unifier ce qui a été divisé. C'est une paire éternelle systole - diastole, syncresis et diachresis de l'univers organique. Dans la Doctrine de couleurs (Farbenlehre), l'idée de la polarité devient un principe opérationnel, les couleurs fondamentales - bleu et jaune-sont des produits de la différentiation par l'ensemble des traits binaires (plus moins).

4. La hiérarchie d'un phénomène naturel est déterminée par la complexité de sa structure. Le trait fondamental des objets inorganiques est l'indifférence des parties à l'égard de leur réunion (Zusammensein). Les corps organiques présentent un autre mode d'être ensemble: les parties se diversifient en organes spécialisés et subordonnés. La totalité organisée 
préserve une forme précise et son équilibre interne. Grâce à la complexité de leur structure, les êtres organiques supérieurs sont non seulement autosuffisants mais ils sont aussi des formations autorégulatrices.

5. Les structures organiques existent dans l'interaction constante avec leur milieu. Les facteurs extérieurs ne peuvent pas influencer les lois et les forces internes de formation propres à l'organisme en question. Ainsi, aux yeux de Goethe, le poisson existe non pas à cause de l'eau, mais il existe dans l'eau et à travers elle, à condition de se trouver dans cet élément intermédiaire dit eau où le poisson non seulement existe mais aussi devient. L'être organique acquiert une adaptation au but (Zweckmässigkeit) pour son milieu, parce qu'il est formé non seulement de l'extérieur mais aussi de l'intérieur. (Cf. Dolezel, 1990: 56-59).

Selon J.-M. Schaeffer, «la spécificité de l’organisme tel que le définissent les romantiques est quadruple»:

«a) L'autonomie: l'organisme possède en lui-même le fondement de son existence et de son évolution. Cette idée est centrale pour l'histoire de la Littérature, puisqu' elle permet de postuler que l'ensemble des faits littéraires forme une totalité autonome, c'est-à-dire que la Littérature contient en elle-même son propre fondement existentiel et évolutif et qu'elle n'est pas soumise à des influences causales externes. Bien entendu, l'unité organique est toujours relative: ce qui est à un niveau d'analyse donné peut être vu comme une unité organique, se révélera à un niveau plus élevé être simplement élément d'un organisme plus englobant; il est ainsi possible de considérer l'œuvre littéraire individuelle à la fois comme un organisme absolument autonome et comme élément d'un ensemble organique plus élevé, que ce soit un genre ou une époque littéraire.

b) L'autodifférenciation avec maintien de l'unité essentielle dans les différentiations: l'unité organique se déploie selon sa propre spontanéité interne en diverses particularisations qui sont interdépendantes et qui toutes se rapportent à l'unité. S'inspirant vaguement de la monadologie leibnizienne, Schlegel va ainsi postuler que les divers éléments d'une unité organique expriment tous la totalité [...].

c) Le concept comme principe de développement: le principe agissant du développement organique n'est autre que son principe spirituel interne, c'est-à-dire son concept [...]. Schlegel affirme [...] que le concept adéquat de la Littérature n'est donné que dans et à travers le développement de l'histoire de la Littérature, en sorte que toute définition abstraite ne saurait qu'être heuristique [...].

d) La téléologie interne. Elle découle directement de l'essentialisme historiciste: les parties procèdent de la totalité conçue comme principe téléologique interne, aussi bien du point de vue formel que du point de vue de l'être-là des concrétisations évolutives. De même que dans la dimension de leur coprésence les parties n'existent que par et pour l'unité organique, dans la dimension de la genèse progressive le déploiement de l'organisme selon des concrétisations historiques successives est entièrement prédéterminé par l'unité originaire. Le principe de téléologie interne exclut toute causalité transitive, extérieure: la finalité évolutive de l'organisme est identique à l'autodéploiement de son essence et il ne peut devenir que ce qu'il est toujours déjà en germe, sans subir la moindre influence extérieure» (Schaeffer, 1992: 145-147).

2. Le terme de "dominante" est introduit par Eikenbaum et repris par Jakobson dans son article de 1935 intitulé "La dominante» ( $c f$. Jakobson, 1977: 77-85). La transcription russe pour ce terme est dominanta.

3. En France, T. Todorov a souligné plusieurs fois les homologies fonctionnelles entre les théories de la langue et de la littérature de ces deux courants à partir des Théories du symbole (op. cit.), mais aussi dans Les Genres du discours (1978), Mikhaïl Bakhtine. Le Principe dialogique (op.cit.), Critique de la critique (op. cit.), ainsi que dans la préface au livre de R. Jakobson, Vie dans le langage. Autoportrait du savant (1982).

4. Une phrase de l'introduction aux textes théoriques du premier romantisme de Lacoue-Labarthe et de Nancy: "l'Athenäum est notre lieu de naissance" atteste l'attitude française vis-à-vis de l'influence effectuée par la doctrine romantique sur l'univers intellectuel occidental. Cette affirmation rencontre la thèse de T. Todorov selon laquelle l'Europe intellectuelle a subi passivement la doctrine romantique pendant près de deux siècles " (Todorov, "Préface» à J.-M. Schaeffer, 1983: 10).
5. En France, la doctrine du premier romantisme allemand est connue surtout grâce à l'étude classique de $\mathrm{R}$. Ayrault (Le Genèse du romantisme allemand, 1961-1976) et grâce à l'étude monumentale de G. Gusdorf (Le Romantisme I. Le savoir romantique et Le Romantisme II. L'homme et la nature, Paris, Payot et Rivages, 1982 et 1983). On trouve l'analyse exhaustive des fondements philosophiques de la doctrine esthétique du premier romantisme dans le livre de J. M. Schaeffer, 1992.

6. Comme le suggèrent également E. Holenstein (dans Jakobson, Gadamer et Holenstein, 1984) et T. Todorov (1977). Sur les rapports romantismeformalisme, voir aussi Hansen-Löve, 1978: I.4 et II; Dolezel, 1990: 53 et 124; Schaeffer, 1983 et 1992, 11-24; 368-375.

7. Ce passage sur l'opposition des projets formaliste et structuraliste résume le contenu du questionnaire proposé à 21 experts russes et occidentaux du formalisme et du structuralisme par les rédacteurs du recueil Sed'myje tynianovskije tchtenija, $\mathrm{n}^{\circ}$ ), Materialy dlja obsujdenija, (Septième colloque de Tynianov, Matériaux pour une discussion, 10-67), publié à Riga et à Moscou en 1995-1996.

8. Nos sources pour ce résumé des théories du romantisme allemand ont été Angelloz, 1980; Ayroux, 1976; Behler, 1992; Todorov, 1977; Die deutsche Literatur in Text und Darstellung, 1991.

9. R. Jakobson, 1971, cf. «index des notions ".

10. En raisonnant sur les principes de choix des «traits qui vont établir l'expansion ", l'alliance linguistique ou l'alliance des langues (jazykovoj sojuz) sur les lois de «l'évolution génétiquement dissemblable, mais structurellement commune des éléments prosodiques", Jakobson écrit: "Le fait de cette sélection, la direction que prend l'expansion et les limites de cette dernière voilà un nœud de problèmes qui doit inciter la linguistique, mais aussi bien les autres disciplines, à progresser, à établir de nouveaux critères dans leur analyse des exemples d'affinités linguistiques secondaires (les Wahlverwandschaft), qui viennent toujours plus nombreux au jour» (1980: 87).

11. Selon D. Combe, "cette "loi", nullement préjudiciable à la littérature selon les Formalistes, repose sur l'idée toute romantique du mélange des genres. L'hybridation des genres qui entraîne leur "décadence" permet à la littérature de se renouveler, à de nouvelles formes de voir le jour" (1992: 116117). Cette grande métaphore organique de la vie et de la mort, de la lutte des formes, de leur épuisement et leur régénération centrale dans l'édifice conceptuel du formalisme, provient donc de la Weltanschaung romantique, où elle constitue un noyau théorique. Dans le champ allemand, la vie, l'instance vitale, se trouve associée à l'Esprit de la grande tradition de la philosophie idéaliste. Ainsi, chez W. von Humboldt, la définition même du langage est liée au travail de l'Esprit qui utilise les sons articulés pour exprimer les idées, le langage étant conçu comme totalité d'actes de parole. Le célèbre dynamisme de la linguistique humboldtienne pose la parole ou le discours cohérent comme la seule forme d'existence de la langue. Ce n'est que dans cet espace du devenir, de la parole émise, qu'existent les mots et les règles de la grammaire. Dans le langage, tout est dynamique et rien n'est statique. La "partie morte» de la langue doit être continûment recréée dans l'Esprit: pour exister la langue doit être parlée et comprise, et c'est ainsi qu'elle passe - dans la totalité de son identité - dans le sujet qui parle cette langue (Jespersen, 1954: 57)

12. C'est en ce sens qu'il faut comprendre l'idée d'O. Brik exposée dans l'article "Rythme et syntaxe» ("Ritm i sintaksis»), à savoir que "le mouvement rythmique précède le vers» et que ce n'est pas le rythme qui peut être compris à partir d'une ligne poétique mais au contraire la ligne qui peut être comprise à partir du mouvement rythmique (Texte der russischen Formalisten, 1972: 164). Ce point de vue de la "totalité dynamique vivante» - à l'opposé des traces déconstructivistes «immanentes" au texte - place le centre de la rythmisation en dehors de la réalisation textuelle. C'est que, théoriquement, toute syllabe peut être accentuée ou non: tout dépend de l'impulsion rythmique. Tout dépend du rythme de la parole versifiée dont le résultat sont ces séquences de syllabes.

La seule et même ligne, la même du point de vue de sa réalisation matérielle - comme dans cet exemple de Brik: "Prolétaires de tous les pays, unissezvous" (Proletarii vsekhstran sojedinjajtes) -, peut être employée dans le langage quotidien, ou encore dans un poème selon ses contraintes rythmiques, mais 
ce sont deux phénomènes différents en dépit de leur identité matérielle et sémantique complète. Dans le premier cas, c'est une phrase formée avec une visée discursive, un slogan politique; dans le deuxième cas, c'est un produit du mouvement rythmique (ibid.: 170). Les formes de l'impulsion rythmique préexistent à la réalisation matérielle. Le mot surgit comme réalisation ou matérialisation de ces formes rythmique (ibid.).

13. Goethe écrit en effet dans le "poème didactique» "Métamorphose des animaux» (1820) : «...car sur un mode double // Elle /la Nature/ fixa la loi suprême, à toute vie assigna des limites [...] // Partant chez l'animal la forme détermine une façon de vivre // Et la façon de vivre, elle, agit en retour puissamment sur les formes. // Telle en sa fixité se montre la figure ordonnée qui incline // Au changement grâce à des êtres agissant sur elle du dehors. // Au dedans toutefois, la force propre aux créatures les plus nobles // Se trouve emprisonnée dans le cercle sacré des figures vivantes. // Ces bornes-là, nul dieu ne les étend et la nature les respecte: // C'est limitée ainsi que la chose parfaite en tout temps fut possible. [...] // Car aucun animal n'a porté de corne au front; et pour cette raison // Il est entièrement impossible à la Mère éternelle de faire // Un lion encorné, quand même elle y mettrait sa puissance totale. // Car il n'est pas en elle assez de masse pour qu'elle puisse implanter // Pleinement les rangées des dents et susciter des cornes et des bois.» (Goethe, 1982: 529-535).

14. Bernstein emprunte cette définition à $\mathrm{B}$. Christiansen dont La Philosophie de l'art (trad. en russe en 1911) est une source fréquentée par les formalistes. Cette vision «intégriste» fonde la critique par Bernstein de l'«analyse objective" du texte poétique qui considère le son comme une construction autonome en dehors de toute corrélation avec la «fonction esthétique» du texte. L'analyse objective, qui aborde le vers du point de vue phonétique, ignore presque entièrement le problème de l'art verbal comme un type particulier de l'art où le facteur phonique acquiert la signification, uniquement en corrélation avec la sémantique et la syntaxe (Texte der russischen Formalisten, 1972: 340).

15. Tynianov écrit dans Le Problème de la langue poétique (1924) : «Le mot n'a pas de signification unique. C'est un caméléon: chaque fois non seulement les nuances diverses, mais aussi parfois des couleurs diverses y surgissent. L'abstraction du "mot" est [...] une sorte de cercle rempli chaque fois de manière nouvelle selon la structure lexicale dans laquelle il entre et les fonctions que porte chacun des éléments discursifs. Il est en quelque sorte la coupe transversale de ces différentes structures lexicales et fonctionnelles [...]. Le mot hors de la proposition n'existe pas. Un mot isolé n'est absolument pas situé dans des conditions hors phrase. Il se trouve seulement dans des conditions autres par rapport au mot de la proposition. En prononçant un mot isolé du "dictionnaire", nous n'obtiendrons pas un "mot en général", un mot purement lexical, mais seulement un mot nouvellement conditionné en comparaison des conditions proposées par le contexte» (Tynianov, 1977: 8182).

Le dualisme entre le " trait principal de la signification" et le "trait secondaire de la signification", posé par Tynianov comme propriété du mot, avance en fait l'idée morphologique d'un fonds immuable - «le trait principal y est toujours présent" (ibid.: 83) - qui assure l'unité du terme à travers ses modifications contextuelles. Le "dynamisme» du mot se manifeste et se trouve conditionné par la multiplicité de ces «traits secondaires de la signification", ces «colorations sémantiques» dont l'ensemble est la "véritable vie " du mot. La dissociation pratiquement complète posée par Tynianov entre les "traits principaux " et les " traits secondaires", de même que l'autonomie entre les aspects matériel et formel, conditionne ce dynamisme extrême où toute métamorphose devient possible.

Tynianov écrit: «Ainsi il y a des lignes d'unité généralisantes permettant au mot d'être reconnu uniquement malgré ses changements occasionnels. Le dualisme peut être considéré comme la division fondamentale des traits de signification en deux classes fondamentales [...]. Ici une remarque préalable: le concept principal ne coïncide pas avec la partie matérielle du mot, de même que le concept de trait secondaire ne coïncide pas avec celui de partie formelle. Le mot [...] est caractérisé par un trait général de signification, le trait principal, mais dans tous les cas il varie très sensiblement en développant ses propres traits (secondaires) de signification" (ibid., 1977: 85-86).

\section{RÉFÉRENCES BIBLIOGRAPHIQUES}

ANGELLOZ, J.-F. [1980]: Le Romantisme allemand, Paris, P.U.F.

AYRAULT, R. [1976] : La Genèse du romantisme allemand, vol. 4, Paris, Aubier. BEHLER, E. [1992]: Le Premier Romantisme allemand, Paris, P.U.F.

COMBE, D. [1992]: Théories du genre, Paris, Hachette.

Die deutshce Literatur in Text und Darstellung, B. 8, Romantik-1, Philipp Reclam jun., Stuttgart, 1991.

DOLEZEL, L. [1990] : Occidental Poetics: Tradiction and Progress, Lincoln and London, University of Nebraska.

EIKHENBAUM, B. [(1925) 1966] : "La théorie de la "méthode formelle" ", dans T. Todorv (sous la dir. de), Théorie de la littérature.

ERLICH, V. [1955] : Russian Formalism, The Hague, Mouton.

EYNDE, L. van [1997]: Introduction au romantisme d'Iéna. Friedrich Schlegel et l'Athenäum, Bruxelles, Ousia.

GOETHE [1982] : Poésie. Du voyage en Italie jusqu'aux derniers poèmes, vol. 2, Paris, Aubier, Montaigne.

HANSEN-LÖVE, A. [1978]: Der russische Formalismus, Methodologische Rekonstruktion seiner Entwicklung aus dem prinzip der Verfremdung, Wien, Verlag der österreichishcen Akademie der Wissenschaften.

HERDER, J. G. [1962]: Idées pour la philosophie de l'histoire de l'humanité, Paris, Aubier-Montaigne.

HOLENSTEIN, E. [1974] : Jakobson ou le structuralisme phénoménologique, Paris, Seghers.

JAKOBSON, R. [1963] : Essais de linguistique générale, Paris, Minuit; [1971]: Selected Writings, vol. 1, Paris, The Hague: [1977]: Huit Questions de poétique, Paris, Seuil; [1980]: Dialogues avec K. Pomorska, Paris, Flammarion.

JAKOBSON, R., H.-G. GADAMER et E. Holenstein [1984] : Das Erbe Hegels II, Francfort-sur-le-Main, Suhrkamp.

JeSPERSEN, O. [1954] : Language, its Nature, Development and Origin, Londres, George Allen.

LACOSTE, J. [1997] : Goethe. Science et philosophie, Paris, P.U.F.

LACOUE-LABARTHE, P. ET J.-L. NANCY [1978] : L'Absolu littéraire. Théorie de la littérature du romanttisme allemand, Paris, Seuil.

MedvedeV, P. et M. BAKHTINE [1928]: La Méthode formelle en science de littérature. Introduction critique à la poétique sociologique, Leningrad.

Sed'myje tynianovskije tchtenija, $\mathrm{n}^{\circ} 9$, Materialy dlja obsujdenija, (Septième colloque de Tynianov, Matériaux pour une discussion, p. 10-67), Riga-Moscou, 1995-1996.

SCHAEFFER, J.-M. [1983] : La Naissance de la littérature. La théorie esthétique du romantisme allemand, Paris, Presses de l'École normale supérieure; [1992] : L'Art de l'age moderne. L'esthétique et la philosophie de l'art du XVIII siècle à nos jours, Paris, Gallimard.

STRIEDTER, J. [1989]: Literary Structure, Evolution, and Value. Russian Formalism and Czech Structuralismus reconsidered, Londres.

TEXTE DER RUSSISCHEN FORMALISTEN [1972], band 2, Wilhelm Fink Verlag München.

Thompson, E. M. [1971]: Russian Formalisme and Anglo-American New Criticism, La Haye-Paris, Mouton.

TODOROV, T. [1977]: Théories du symbole, Paris, Seuil; [1981]: Mickhaïl Bakhtine. Le principe dialogique, Paris, Seuil; [1984]: Critique de la critique, Paris, Seuil; (sous la dir. de) [1966] : Théorie de la littérature, Paris, Seuil.

TYNIANOV, I. [1977]: Le Vers lui-même. Les problèmes du vers, Paris, Union générale d'éditions.

WaChSMUTH, A.B. [1966]: Geeinte zwienatur. Aufsätze zu Goethes naturwissenschftlichem Denken, Berlin und Weimar, Aufbau-Verlag.

ZIRMUNDSKIJ, V. [1996] : Nemezkij romantism i sovremennaja mistika (Le Romantisme allemandet la mystique contemporaine), Saint-Pétersbourg, Axioma. 